\title{
Análisis multivariado de indicadores zoométricos de madurez sexual de gallinas reproductoras Campero INTA
}

\author{
Sanz, P.'; Sindik, M.'; Fernández, R.'; Revidatti, F.'; Canet, Z. ${ }^{2,3}$ \\ ${ }^{1}$ Cátedra Producción de Aves, Fac.Cs.Vet. UNNE, Corrientes (Argentina). \\ ${ }^{2}$ Cát.Genética, Fac.Cs.Vet. UNR. ${ }^{3}$ INTA Pergamino. \\ E-mail: granja@vet.unne.edu.ar
}

\section{Resumen}

Sanz, P.; Sindik, M.; Fernández, R.; Revidatti, F.; Canet, Z.: Análisis multivariado de indicadores zoométricos de madurez sexual de gallinas reproductoras Campero INTA. Rev. Vet. 32: 1, 43-47, 2021. El objetivo del presente estudio fue evaluar el comportamiento de los indicadores zoométricos involucrados en el proceso biológico de la madurez sexual mediante análisis multivariado, para explicar su comportamiento conjunto y caracterizar su desarrollo. Se trabajó con 400 gallinas pertenecientes a 2 genotipos (sintética E y el híbrido ES*A) que recibieron dos planos nutricionales (control y tratado) entre las semanas 5 y 22. Se realizó análisis canónico para representar la asociación lineal de las variables, obteniendo ecuaciones que permiten explicar una porción de la variancia total de los datos independiente de las restantes componentes canónicas. Se generaron cuatro componentes canónicas de las semanas 22 y 24. Los resultados de la semana 22 arrojaron que las dos primeras componentes explican el $94,87 \%$ de la variación observada. La primera componente (PCC) explica el $81,86 \%$ de la variancia y se correlaciona en forma positiva y significativa con $\mathrm{ICr}(\mathrm{r}=0,787$; $p<0,0001), A C a(r=0,626 ; p<0,0001)$, LAb $(r=0,527 ; p=0,0001)$ y no muestra asociación significativa con ancho $\mathrm{ACl}(\mathrm{r}=-0,072 ; \mathrm{p}=0,268)$, correlacionando principalmente con el índice de cresta. La segunda componente (SCC) explica el $13,01 \%$ de la variancia y se correlaciona en forma positiva y significativa con las cuatro variables, pero en mayor medida con $\mathrm{ACl}$ $(\mathrm{r}=0,921 ; \mathrm{p}=0,0001)$ y menor medida con $\mathrm{ICr}(\mathrm{r}=0,233 ; \mathrm{p}=0,0003), \mathrm{ACa}(\mathrm{r}=0,572 ; \mathrm{p}<0,0001)$ y LAb $(r=0,515 ; p<0,0001)$. Los resultados de la semana 24 para las PCC explica el $70,41 \%$ de la variancia y correlaciona en forma positiva y significativa con el $\operatorname{ICr}(r=0,982 ; p<0,0001)$, el $\mathrm{ACl}(\mathrm{r}=0,615 ; \mathrm{p}<0,0001), \mathrm{ACa}(\mathrm{r}=0,423 ; \mathrm{p}<0,0001)$ y LAb $(\mathrm{r}=0,459 ; \mathrm{p}<0,0001$, haciéndolo principalmente con el índice de cresta. La SCC explica el $26,98 \%$ de la variancia y correlaciona, en forma positiva y significativa con el $\mathrm{ACl}(\mathrm{r}=0,525 ; \mathrm{p}<0,0001), \mathrm{ACa}(\mathrm{r}=0,887$; $p<0,0001)$ y LAb $(r=0,671 ; p<0,0001)$, correlacionando principalmente con el ancho de cadera y sin mostrar asociación con el $\mathrm{ICr}(\mathrm{r}=-0,017 ; \mathrm{p}=0,799)$. Esto implica que, a mayor valor de PCC las aves presentan esencialmente mayor índice de cresta, ancho de cloaca, ancho de cadera y longitud abdominal. Se concluye que el aumento en la asignación de nutrientes produce un aumento significativo en el valor promedio de la componente canónica, lo que sugiere un adelanto de la madurez sexual tanto en E como en ES*A de mayor magnitud en las híbridas.

Palabras clave: gallinas, desarrollo, recría, reproducción, restricción.

\begin{abstract}
Sanz, P.; Sindik, M.; Fernández, R.; Revidatti, F.; Canet, Z.: Multivariate analysis of zoometric indicators of sexual maturity of breeding hens Campero INTA. Rev. Vet. 32: 1, $43-47,2021$. The aim of the present study was to evaluate the behavior of the zoometric indicators involved in the biological process of sexual maturity through multivariate analysis to explain their joint behavior and characterize their development. We worked with 400 pullets belonging to 2 genotypes (synthetic $\mathrm{E}$ and the hybrid $\mathrm{ES}^{*} \mathrm{~A}$ ) that received two nutritional levels (control and treated) between weeks 5 and 22. Canonical analysis was performed to represent the linear association of the variables obtaining equations that allow explaining a portion of the total variance of the data independent of the remaining canonical components. Four canonical components were generated from week 22 and 24. The results of the week 22 showed that the first two components explain $94.87 \%$ of the observed variation. The first component (PCC) explains $81.86 \%$ of the variance and is positively and significantly correlated with comb index (ICr) $(\mathrm{r}=0.787 ; \mathrm{p}<0.0001)$, hip width (ACa) $(\mathrm{r}=0.626 ; \mathrm{p}<0.0001)$, abdominal length $(\mathrm{LAb})(\mathrm{r}=0.527 ; \mathrm{p}=0.0001)$ and shows no significant association with cloa-
\end{abstract}


cal width ( $\mathrm{ACl}, \mathrm{r}=-0.072 ; \mathrm{p}=0.268$ ), correlating mainly with comb index. The second component (SCC) explains $13.01 \%$ of the variance and is positively and significantly correlated with the four variables, but to a greater extent with $\mathrm{ACl}(\mathrm{r}=0.921 ; \mathrm{p}=0.0001)$ and to a lesser extent with $\mathrm{ICr}(\mathrm{r}=0.233 ; \mathrm{p}=0.0003)$, ACa $(\mathrm{r}=0.572 ; \mathrm{p}<0.0001)$ and $\mathrm{LAb}(\mathrm{r}=0.515 ; \mathrm{p}<0.0001)$. The results for week 24 for the PCC explain $70.41 \%$ of the variance and correlate in a positive and significant way with the $\operatorname{ICr}(r=0.982 ; \mathrm{p}<0.0001)$, the $\mathrm{ACl}(\mathrm{r}=0.615 ; \mathrm{p}<0.0001), \mathrm{ACa}$ $(\mathrm{r}=0.423 ; \mathrm{p}<0.0001)$ and LAb $(\mathrm{r}=0.459 ; \mathrm{p}<0.0001$, doing it mainly with the comb index. The SCC explains $26.98 \%$ of the variance and correlates, in a positive and significant way with the $\mathrm{ACl}(\mathrm{r}=0.525 ; \mathrm{p}<0.0001), \mathrm{ACa}(\mathrm{r}=0.887 ; \mathrm{p}<0.0001)$ and $\mathrm{LAb}(\mathrm{r}=0.671 ; \mathrm{p}<0.0001)$, mainly correlating with hip width and does not show an association with the $\operatorname{ICr}(r=-0.017 ; \mathrm{p}=0.799)$. This implies that, the higher the CCP value, the birds have essentially a higher comb index, cloacal width, hip width and abdominal length It is concluded that the increase in the allocation of nutrients produces a significant increase in the average value of the canonical component, which suggests an advance of sexual maturity in both $\mathrm{E}$ and $\mathrm{ES}^{*} \mathrm{~A}$ of greater magnitude in hybrids.

Key words: hens, development rearing, reproduction, restriction.

\section{INTRODUCCIÓN}

El esquema general de la fisiología endocrina de la reproducción en la gallina está sujeto a variaciones derivadas de la selección genética y de los efectos ambientales.

La selección divergente para crecimiento y reproducción, llevó a la obtención de líneas y estirpes pesadas para producción de carne que presentan una marcada correlación negativa entre aptitud reproductiva y peso corporal, lo que hace necesario desarrollar estrategias de manejo efectivas que permitan compatibilizar ambos objetivos en las reproductoras.

De la misma forma se han encontrado diferencias en la aptitud reproductiva entre genotipos para carne seleccionados para crecimiento o para características relacionadas con la aptitud reproductiva ${ }^{3}$. Las señales ambientales proveen información sobre la temporada de reproducción. Éstas disparan cambios neuroanatómicos, neuroendocrinos y fisiológicos, preparando a los animales para la reproducción ${ }^{1}$.

La baja eficiencia reproductiva de las gallinas reproductoras para carne lleva a que las prácticas de manejo actuales incluyan restricciones alimenticias a menudo imprecisas y muy severas para limitar el crecimiento corporal en un esfuerzo para mejorar la producción de huevos ${ }^{8}$.

Los diferentes programas de manejo (alimentación, luz, densidad, métodos de crianza y otros) están diseñados para modular la expresión del potencial de crecimiento y mejorar la eficiencia en la producción de huevos fértiles incubables ${ }^{6}$. La aptitud reproductiva de una población es modificada por múltiples factores que provienen del ambiente e interactúan entre sí contribuyendo a incrementar la capacidad de los reproductores pesados.

Si bien se han realizado avances significativos mediante el diseño de programas de luz y alimentación, aún deben desarrollarse programas de manejo especiales adaptados a las condiciones y recursos de cada caso en particular, estando en proceso de evolución continua siguiendo a los cambiantes requerimientos de las aves ${ }^{7}$.

El efecto de la alimentación inadecuada sobre la producción de hormonas se ve reflejado en alteraciones del desarrollo folicular, tanto durante las etapas previas a la madurez sexual como en el ave adulta, provocando disrupciones en el patrón normal de producción o secreción del factor de liberación hipotalámico ${ }^{1}$.

Adicionalmente, los programas de manejo aplicados durante la cría y la recría pueden modificar en forma marcada las condiciones en las que se produce la madurez sexual, lo que hace necesario evaluar este proceso en sus aspectos anatómicos, fisiológicos y reproductivos. El proceso de maduración sexual en la gallina está asociado a un incremento en los niveles plasmáticos de 17 ß-estradiol.

Esta variable es utilizada en el laboratorio para monitorear el grado de maduración de las aves, método que -aunque confiable- es invasivo y poco práctico. Los estrógenos producen cambios fácilmente perceptibles en los caracteres sexuales secundarios, lo cual permite que sean utilizados con mayor facilidad para evaluar la madurez sexual

Debido a que el tamaño de la cresta correlaciona positivamente con los niveles de estrógenos y con la edad al primer huevo, dicha variable es considerada útil para medir la tasa de maduración sexual. Hacia el final de la recría, la cresta aumenta de volumen, se torna más turgente y el ave puede perder algunas plumas primarias (muda prenupcial). Además, los huesos púbicos se ensanchan para dar paso al huevo.

Normalmente después de 10 a 11 días de iniciadas estas señales de pubertad perceptibles, se pone el primer huevo. El tamaño de la cresta comienza a incrementarse 3 a 5 semanas antes de la puesta del primer huevo; algunos autores recomiendan medir su altura a nivel de la cuarta espiga ${ }^{4}$

El inicio de la madurez sexual también se puede determinar mediante el examen de la cloaca en sus aspec- 
tos cualitativos y cuantitativos: hembras sexualmente maduras tienen $2,5 \mathrm{~cm}$ de ancho de cloaca ${ }^{10}$.

El objetivo del presente estudio fue evaluar el comportamiento de los indicadores zoométricos involucrados en el proceso biológico de la madurez sexual mediante el análisis multivariado para explicar su comportamiento conjunto y caracterizar su desarrollo.

\section{MATERIAL Y MÉTODOS}

Las distintas etapas del ciclo de los reproductores se llevaron a cabo en el Centro de Multiplicación de Aves de la Estación Experimental Agropecuaria Corrientes del INTA, en la Ruta Nacional $\mathrm{N}^{\circ} 12, \mathrm{~km} 1008$, El Sombrero (Corrientes, Argentina).

En el ensayo se trabajó con 200 gallinas pertenecientes a la población sintética materna $\mathrm{E}$ del pollo Campero INTA (composición genética teórica $50 \%$ Cornish Colorada; 50\% Rhode Island Colorada) y 200 al híbrido simple producto del cruzamiento entre las poblaciones sintéticas maternas ES*A (composición genética teórica 81,25\% Cornish Colorada; $18,75 \%$ Rhode Island Colorada).

Las aves de cada genotipo se subdividieron en dos grupos, cada uno de los cuales recibió entre las semanas 5 y 22 uno de los siguientes tratamientos que consisten en modificaciones cuantitativas de las dietas: Grupo Estándar (programa de alimentación restringida, habitualmente aplicado en el establecimiento) y Grupo Estándar+10 (programa de alimentación con una modificación cuantitativa de la dieta consistente en un aumento del $10 \%$ en el aporte de nutrientes respecto del grupo control).

En las semanas 22 y 24 del ciclo de las reproductoras se registró individualmente en cada ave: índice de cresta (ICr, producto del alto de cresta desde su base hasta la punta de la cuarta espiga por su longitud desde el extremo anterior hasta el posterior, ambos registrados con calibre en $\mathrm{mm}^{2}$.), ancho de cloaca ( $\mathrm{ACl}$, distancia transversal en $\mathrm{mm}$ existente entre ambos extremos laterales de la cloaca con el ave en decúbito dorsal), ancho de la cadera (ACa, distancia transversal en $\mathrm{mm}$ existente entre ambos isquiones con el ave en decúbito dorsal) y longitud abdominal (LAb, distancia longitudinal en mm existente entre la apófisis esternal caudal y una línea imaginaria que une ambos extremos de los huesos coxales con el ave en decúbito dorsal).

Se realizó análisis canónico para representar la asociación lineal de las variables obteniendo ecuaciones que permiten explicar una porción de la variancia total de los datos independiente de las restantes componentes canónicas ${ }^{2}$.

\section{RESULTADOS Y DISCUSIÓN}

En la Tabla 1 y el Gráfico 1 se observan los resultados obtenidos al final de la semana 22 para los distintos grupos experimentales y su significancia estadística.
Se generaron cuatro componentes canónicas (las dos primeras explican el $94,87 \%$ de la variancia) cuyas ecuaciones asociadas son:

Primera Componente Canónica $(\mathrm{PCC})=0,0027 \mathrm{ICr}-$ $0,1908 \mathrm{ACl}+0,9655 \mathrm{ACa}+0,3714 \mathrm{LAb}$

Segunda Componente Canónica $(\mathrm{SCC})=-0,00077 \mathrm{ICr}$ $+0,2331 \mathrm{ACl}+0,2394 \mathrm{ACa}+0,5150 \mathrm{LAb}$

La primera componente (PCC) explica el $81,86 \%$ de la variancia y se correlaciona en forma positiva y significativa con $\mathrm{ICr}(\mathrm{r}=0,787 ; \mathrm{p}<0,0001)$, ACa $(\mathrm{r}=0,626$; $\mathrm{p}<0,0001), \mathrm{LAb}(\mathrm{r}=0,527 ; \mathrm{p}=0 ; 0001)$ y no muestra asociación significativa con $\mathrm{ACl}(\mathrm{r}=-0,072 ; \mathrm{p}=0,268)$, correlacionando principalmente con el índice de cresta. Esto implica que, a mayor valor de PCC las aves presentan esencialmente mayor índice de cresta, ancho de cadera y longitud abdominal.

La segunda componente (SCC) explica el 13,01\% de la variancia y se correlaciona en forma positiva $y$ significativa con las cuatro variables, pero en mayor medida con $\mathrm{ACl}(\mathrm{r}=0,921 ; \mathrm{p}=0,0001)$ y menor medida con $\mathrm{ICr}(\mathrm{r}=0,233 ; \mathrm{p}=0,0003), \mathrm{ACa}(\mathrm{r}=0,572 ; \mathrm{p}<0,0001)$ y LAb $(r=0,515 ; p<0,0001)$. Es decir, que a mayor valor de SCC las aves presentan básicamente mayor ancho de cloaca y menor índice de cresta, longitud abdominal y ancho de cadera.

La inspección de los gráficos de barras correspondientes a la PCC muestra que bajo el régimen estándar de asignación de nutrientes, las aves E (E Est: 3,185 \pm 0,1377 ) presentan mayor valor de la primera componente al igual que cuando se las compara en un ambiente con un aporte agregado del 10\% (E +10: 3,617 $\pm 0,1487$ ), que las aves del genotipo ES*A (ES*A Est: $1,470 \pm 0,1026$ y ES*A +10: 2,169 $\pm 0,1166)$.

En la Tabla 2 y Gráfico 2 se exponen los resultados obtenidos en la semana 24 para los distintos grupos experimentales y su significancia estadística.

Se generaron cuatro componentes canónicas (las dos primeras explican el $97,39 \%$ de la variancia) cuyas ecuaciones asociadas son:

$1^{\circ}$ Componente Canónica $(\mathrm{PCC})=0,0024 \mathrm{ICr}+0,0472$ $\mathrm{ACl}-0,3635 \mathrm{ACa}+0,3269 \mathrm{LAb}$

$2^{\circ}$ Componente Canónica $(\mathrm{SCC})=-0,0013 \mathrm{ICr}+0,0342$ $\mathrm{ACl}+1,2918 \mathrm{ACa}+0,1388 \mathrm{LAb}$

La PCC explica el $70,41 \%$ de la variancia y correlaciona en forma positiva y significativa con el $\mathrm{ICr}$ $(r=0,982 ; p<0,0001)$, el ACl $(r=0,615 ; p<0,0001), \mathrm{ACa}$ $(\mathrm{r}=0,423 ; \mathrm{p}<0,0001)$ y LAb $(\mathrm{r}=0,459 ; \mathrm{p}<0,0001$, haciéndolo principalmente con el índice de cresta.

Esto implica que, a mayor valor de PCC, las aves presentan esencialmente mayor índice de cresta, ancho de cloaca, ancho de cadera y longitud abdominal. La SCC explica el $26,98 \%$ de la variancia y correlaciona, en forma positiva y significativa con el $\mathrm{ACl}(\mathrm{r}=0,525$; 
Tabla 1. Primera y segunda componentes canónicas derivadas del análisis discriminante aplicado a cuatro indicadores zoométricos de madurez sexual en dos genotipos de reproductoras camperas bajo dos regímenes de asignación de nutrientes.

\begin{tabular}{lcccc}
\hline & \multicolumn{2}{c}{ sintética E } & \multicolumn{2}{c}{ híbrido ES x A } \\
\cline { 2 - 5 } & estándar & estándar $+\mathbf{1 0 \%}$ & estándar & estándar $+10 \%$ \\
\hline PCC & $3,185 \pm 0,1377$ & $3,617 \pm 0,1487$ & $1,470 \pm 0,1026$ & $2,169 \pm 0,1166$ \\
SCC & $7,661 \pm 0,1032$ & $8,417 \pm 0,1522$ & $7,938 \pm 0,0986$ & $8,513 \pm 0,1282$ \\
\hline
\end{tabular}

Todos los valores corresponden a la media aritmética \pm error estándar.

Tabla 2. Primera y segunda componentes canónicas derivadas del análisis discriminante aplicado a cuatro indicadores zoométricos de madurez sexual en dos genotipos de reproductoras camperas, bajo dos regímenes de asignación de nutrientes.

\begin{tabular}{lcccc}
\hline & \multicolumn{2}{c}{ sintética E } & \multicolumn{2}{c}{ húbrido ES x A } \\
\cline { 2 - 5 } & estándar & estándar $+\mathbf{1 0 \%}$ & estándar & estándar $+10 \%$ \\
\hline PCC & $4,181 \pm 0,1671$ & $4,652 \pm 0,1551$ & $2,925 \pm 0,0803$ & $3,130 \pm 0,0814$ \\
SCC & $6,181 \pm 0,1411$ & $7,227 \pm 0,1169$ & $6,628 \pm 0,1269$ & $7,223 \pm 0,1342$ \\
\hline
\end{tabular}

Todos los valores corresponden a la media aritmética \pm error estándar.

$\mathrm{p}<0,0001), \mathrm{ACa}(\mathrm{r}=0,887 ; \mathrm{p}<0,0001)$ y LAb $(r=0,671 ;$ $\mathrm{p}<0,0001)$, correlacionando principalmente con el ancho de cadera y no muestra asociación con el $\mathrm{ICr}(\mathrm{r}=$ $-0,017 ; \mathrm{p}=0,799)$.

La inspección de los gráficos de barras correspondientes a la PCC muestra que bajo el régimen estándar de asignación de nutrientes, las aves E (E Est: 4,181 \pm $0,1671)$ presentan mayor valor de la primera componente al igual que cuando se las compara en un ambiente con un aporte agregado del 10\% (E+10:4,652 $\pm 0,1551)$, que las aves del genotipo ES*A (ES*A Est: $2,925 \pm$ $0,0803$ y $\mathrm{ES} * \mathrm{~A}+10: 3,130 \pm 0,0814)$.
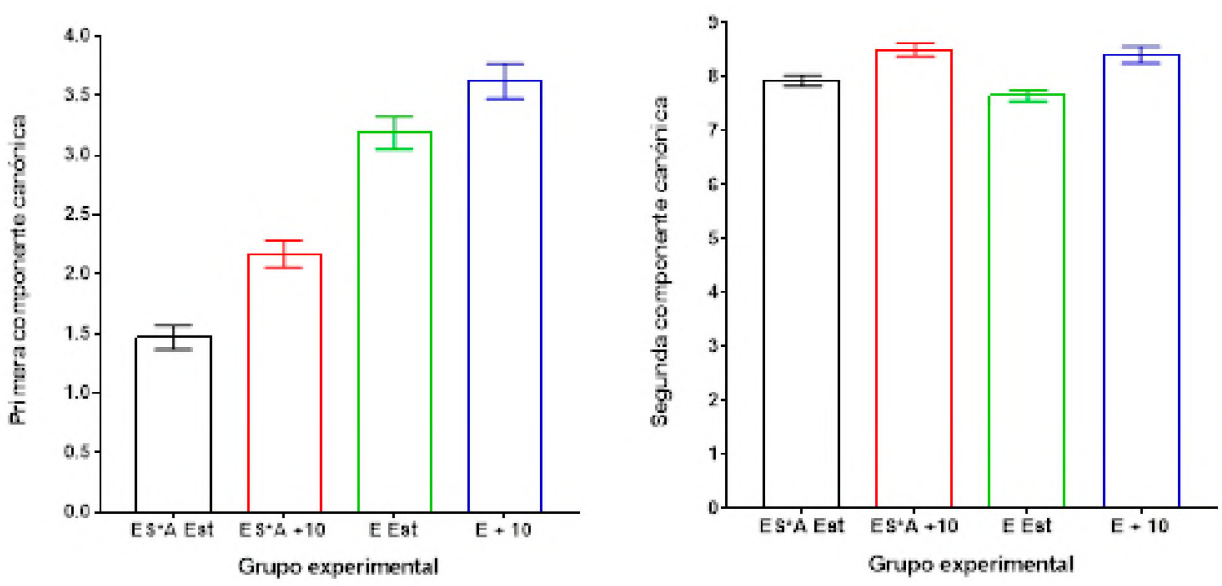

Gráfico 1. Grupos de barras primera y segunda componentes canónicas derivadas del análisis discriminante aplicado a cuatro indicadores zoométricos de madurez sexual en 2 genotipos de reproductoras camperas bajo 2 regímenes de asignación de nutrientes
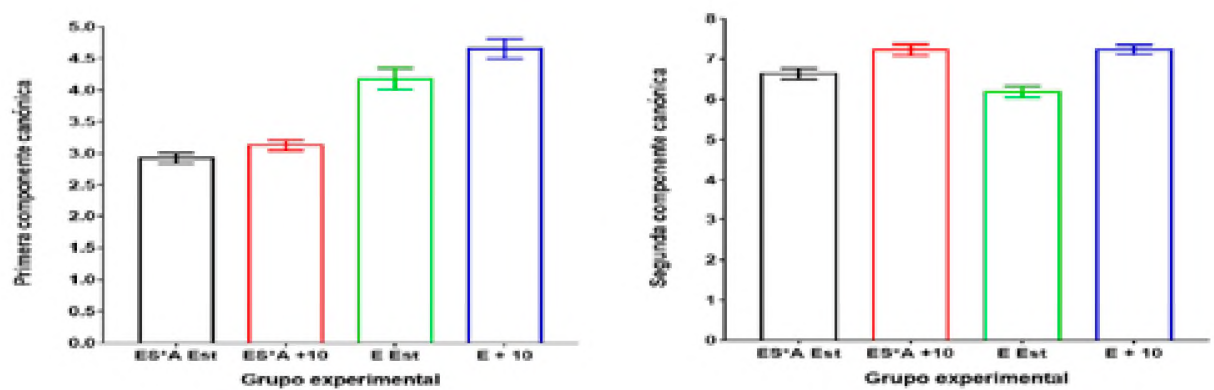

Gráfico 2: Gráfico de barras de primera y segunda componentes canónicas derivadas del análisis discriminante aplicado a cuatro indicadores zoométricos de madurez sexual en dos genotipos de reproductoras camperas bajo dos regímenes de asignación de nutrientes. 
La PCC discrimina grupos genéticos y la SCC por el nivel de asignación de nutrientes. Las aves con un $10 \%$ más de asignación presentan un mayor desarrollo de los indicadores zoométricos de madurez sexual en ambos genotipos.

El mayor valor de los caracteres que exhibe la PCC indica un ave más cercana a la madurez sexual, lo cual permite asumir que al comparar dos genotipos en el mismo ambiente, el de mayor valor promedio de PCC está más cerca de la madurez sexual, asociada con un mayor índice de cresta, ancho de cloaca, ancho de cadera y longitud abdominal.

A su vez manteniendo los genotipos bajo dos ambientes diferentes, el de mayor valor promedio de PCC está más cerca de la madurez sexual, por lo cual la componente en sí indicaría mayor madurez. Hallazgos que coinciden con autores ${ }^{9}$ señalan que un aumento en la asignación de alimento hacia el final de la recría estimula una maduración sexual más uniforme en el lote debido a que existe una declinación de los requerimientos para crecimiento junto a un incremento de las demandas para el desarrollo del aparato reproductor e inicio de la producción de huevos.

Los resultados alcanzados por otros autores repor$\tan$ que las aves sexualmente maduras alcanzan aproximadamente $25 \mathrm{~mm}$ de ancho de cloaca, sugiriendo su medición como método práctico para la evaluación de la madurez sexual ${ }^{9}$.

A mayor valor de SCC las aves presentan básicamente menor índice de cresta y mayor ancho de cadera, longitud abdominal y ancho de cloaca. Este resultado muestra que existen dos fuentes de variación para el índice de cresta: una predominante, asociada positivamente al ancho de cloaca y la longitud abdominal y prácticamente independiente del ancho de cadera, y una segunda, menos trascendente, asociada negativamente con los tres indicadores restantes.

Tales resultados coinciden con otros estudios que asignan un fuerte componente genético a las variables relacionadas a la estructura ósea de la pelvis, con fuerte repercusiones en la fertilidad ${ }^{5}$.

Se concluye que el aumento en la asignación de nutrientes produce un aumento significativo en el valor promedio de la componente canónica, lo cual sugiere un adelanto de la madurez sexual tanto en E como en $\mathrm{ES}^{*} \mathrm{~A}$, de mayor magnitud en las híbridas.

\section{REFERENCIAS}

1. Akins CH, Burns M. 2001. Visual control of sexual behavior. http://www.pigeon. psy.tufts.edu/avc/akins/-115k.

2. Carrasco JL, Hernán MA. 1993. Estadistica multivariante en las ciencias de la vida. Ed. Ciencia 3, Madrid.

3. Decuypere E, Bruggeman V, Onagbesan O, Safi $M$. 1999. Endocrine physiology of reproduction in the female chicken: old wine in new bottles. Proceedings of the International Congress on Bird Reproduction, Tours Fr 145153.

4. Joseph NS, Robinson FE, Renema RA, Thorsteinson KA. 2003. Comb growth during sexual maturation in female broiler breeders. J Appl Poult Res 12: 7-13.

5. McGary S, Estevez I, Bakst M. 2003. Potential relationships between physical traits and male broiler breeder fertility. Poultry Science 82: 328-337.

6. Mendoza AM. 2006. Nutrient utilization and reproductive performance in broiler breeder hens. A thesis submitted to the Graduate Faculty of the University of Georgia in partial fulfillment of the requirements for the degree Master of Science. University of La Salle, Colombia, $95 \mathrm{p}$.

7. Poole R. 1974. Sampling and the estimation of population parameters. An introduction to quantitative ecology, Ed. McGraw Hill, New York, p. 292-324.

8. Ramachandran R. 2014. Current and future reproductive technologies for avian species. Adv Exp Med Biol 752: 2331.

9. Renema RA, Rustad ME, Robinson FE. 2007. Implications of changes to commercial broiler and broiler breeder body weight targets over the past 30 years. World's Poultry Science Journal 63: 457- 472.

10. Wright $\mathbf{D}$ et al. 2012. Onset of sexual maturity in female chickens is genetically linked to loci associated with fecundity and a sexual ornament. Reproduction in domestic animals 47: 31-36. 